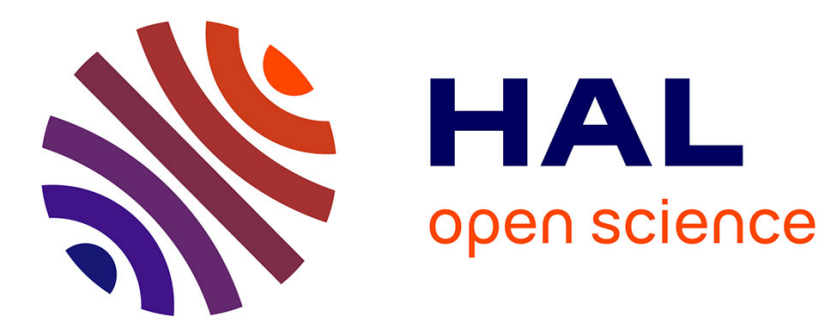

\title{
Acquis et objectifs de la recherche clinique sur le cancer du rectum
}

Philippe Maingon, Jean-Marc Simon, Charles-Henri Canova, Idriss Troussier, Nadia Besson, Elise Caillot, Huguet F.

\section{- To cite this version:}

Philippe Maingon, Jean-Marc Simon, Charles-Henri Canova, Idriss Troussier, Nadia Besson, et al.. Acquis et objectifs de la recherche clinique sur le cancer du rectum. Cancer/Radiothérapie, 2017, 21 (6-7), pp.533 - 535. 10.1016/j.canrad.2017.07.019 . hal-01620212

\section{HAL Id: hal-01620212 \\ https://hal.sorbonne-universite.fr/hal-01620212}

Submitted on 20 Oct 2017

HAL is a multi-disciplinary open access archive for the deposit and dissemination of scientific research documents, whether they are published or not. The documents may come from teaching and research institutions in France or abroad, or from public or private research centers.
L'archive ouverte pluridisciplinaire HAL, est destinée au dépôt et à la diffusion de documents scientifiques de niveau recherche, publiés ou non, émanant des établissements d'enseignement et de recherche français ou étrangers, des laboratoires publics ou privés. 


\section{Acquis et objectifs de la recherche clinique sur le cancer du rectum}

\section{Clinical research for rectal carcinoma: State of the art and objectives}

Philippe Maingon ${ }^{1}$, Jean-Marc Simon², Charles-Henri Canova ${ }^{2}$, Idriss Troussier ${ }^{2}$, Nadia Besson ${ }^{3}$, Elise Caillot ${ }^{3}$, Florence Huguet ${ }^{3}$

1- UPMC Sorbonne Université, Service d'Oncologie Radiothérapie, GHU La Pitié Salpêtrière Charles Foix, 47/83 Bd de l’Université, 75013 Paris

2- Service d'Oncologie Radiothérapie, GHU La Pitié Salpêtrière Charles Foix, 47/83 Bd de l'Université, 75013 Paris

3- UPMC Sorbonne Université, Service d'Oncologie Radiothérapie, Hôpital Tenon, 20 rue de la Chine, 75020 Paris

Corresponding author : Pr. Philippe MAINGON, Service d'Oncologie Radiothérapie, GHU La Pitié Salpêtrière Charles Foix, 47/83 Bd de l'Université, 75013 Paris, +33184827276, philippe.maingon@aphp.fr 
Le traitement des cancers du rectum repose sur une prise en charge pluridisciplinaire et le plus souvent sur une approche multimodale intéressant la gastro-entérologie, l'oncologie-médicale, l'oncologie-radiothérapie et la chirurgie. Les différents objectifs qui doivent être discriminés dépendent des caractéristiques de la tumeur. Le challenge de la prise en charge des petites tumeurs repose sur la conservation d'un sphincter fonctionnel sans risque de récidive locale. Le traitement standard des maladies localement avancées vise, par ordre de priorité, à guérir le malade en minimisant les séquelles tardives de la prise en charge. Chacune de ces situations cliniques se trouve désormais démembrée grâce aux progrès de la chirurgie et d'une approche personnalisée au patient à la caractéristique clinique de la maladie. Elles sont désormais l'objet d'études thérapeutiques spécifiques.

Mots clés : Cancer du rectum, recherche clinique, radiothérapie, radio-chimiothérapie 


\begin{abstract}
The treatment of rectal carcinoma is based on pluridisciplinary strategy and multimodal approaches including gastro-intestinal tract specialists, medical oncologists, radiation oncologists and surgery. The different objectives should be declined according to the characteristics of the tumors. The aim of the therapist would be to select the best strategy offering to the patient to be cured with as less as possible late adverse toxicity. The challenge associated to the treatment of small tumors is to maintain a functional anal sphincter while minimizing the risk of local recurrence. The standard treatment of locally advanced disease is aiming firstly to cure the patient secondly to prevent late complications. Each of these clinical presentations of the disease has to be considered as a whole taking into account the new surgical techniques and a personalized approach adapted to the tumor. Nowaday they should be studied with dedicated clinical trials.
\end{abstract}

Keywords: Radiotherapy, radiation-oncology, combined modality treatment, chemoradiation, clinical research 


\section{INTRODUCTION}

Le traitement des cancers du rectum repose sur une prise en charge pluridisciplinaire et le plus souvent sur une approche multimodale intéressant la gastro-entérologie, l'oncologie-médicale, l'oncologie-radiothérapie et la chirurgie. A ces spécialistes vient s'ajouter, dans l'arbre décisionnel de la prise en charge des traitements, les spécialistes en imagerie, les radio-diagnosticiens ou spécialistes en médecine nucléaire, les pathologistes et bientôt les biologistes moléculaires. Les différents objectifs qui doivent être discriminés dépendent des caractéristiques de la tumeur (sa taille, sa localisation par rapport au sphincter, ses critères d'agressivité et son extension locorégionale). La mission des thérapeutes va être de proposer la meilleure stratégie offrant au patient la possibilité de guérir de la tumeur avec un minimum de séquelles. Le challenge de la prise en charge des petites tumeurs repose sur la conservation d'un sphincter fonctionnel sans risque de récidive locale. Le traitement standard des maladies localement avancées vise, par ordre de priorité, à guérir le malade en minimisant les séquelles tardives de la prise en charge. Chacune de ces situations cliniques se trouve désormais démembrée grâce aux progrès de la chirurgie et d'une approche personnalisée au patient à la caractéristique clinique de la maladie. Il est possible, même pour des maladies localement avancées, de personnaliser la prise en charge thérapeutique, notamment à partir de l'évaluation précoce de la réponse tumorale à un traitement d'induction. A chaque problématique clinique correspond désormais un spectre de protocole d'évaluation adaptée.

\section{TUMEURS LOCALISEES DE BON PRONOSTIC}

Le traitement standard $\mathrm{du}$ cancer $\mathrm{du}$ rectum est l'exérèse rectale précédée d'une radiochimiothérapie néo-adjuvante chez les patients à risques de récidive locale. La chirurgie d'exérèse complète du mésorectum est associée à une morbidité qui altère la qualité de vie. La radiochimiothérapie, si elle entraine une réponse tumorale complète, rend la chirurgie extensive inutile. Les résultats de l'essai GRECCAR 2 suggère, pour les tumeurs rectales T2, T3 sélectionnées, qu'une exérèse locale puisse être une alternative raisonnable à la chirurgie radicale [8]. Cet essai a montré qu'une réponse histologique complète est plus fréquente en cas de petites tumeurs (40\% pour les tumeurs classées T2, T3, inférieures à $4 \mathrm{~cm}$ ). Ces patients bons répondeurs n'ont pas présenté de métastase ganglionnaire dans le mésorectum. Enfin, cet essai a confirmé l'excellente valeur prédictive négative de l'imagerie par résonance magnétique (IRM) initiale des patients cliniquement N0. Elle permet de confirmer que l'exérèse locale est une bonne alternative à la chirurgie radicale.

La préservation d'organe pour les cancers du rectum comporte, soit l'option observationnelle, soit la tumorectomie trans-anale. La stratégie observationnelle proposée par l'équipe d'Habr-Gamar est difficile à reproduire en raison de la faible sensibilité de l'imagerie pour affirmer la réponse complète [5,9]. A l'opposé, la tumorectomie permet de bénéficier de l'examen anatomopathologique de la pièce opératoire afin de s'assurer de la réalité de la réponse tumorale et de la quantifier. Une mauvaise réponse constatée sur l'examen définitif permet de recommander une exérèse rectale radicale complémentaire. 
La question pertinente actuelle est d'optimiser la réponse au traitement néo-adjuvant afin d'augmenter le taux de réponse pathologique complète permettant de proposer une exérèse rectale limitée. Les solutions permettant d'améliorer les résultats basés sur ce critère principal sont de modifier la chimiothérapie concomitante à la radiothérapie ou de proposer une chimiothérapie néo-adjuvante avant la radio-chimiothérapie, d'augmenter la dose de radiothérapie, d'introduire une curiethérapie de complément. L'addition de l'Oxaliplatine à la Capécitabine concomitante à la radiothérapie est associée à une augmentation de la toxicité sans bénéfice sur la réponse tumorale ni avantage sur la survie $[1,2,4]$. L'apport des thérapies ciblées comme des anti-angiogéniques a été décevant $[3,6]$. La chimiothérapie néo-adjuvante avant la radio-chimiothérapie a été introduite pour les tumeurs localement avancées dans l'étude GRECCAR 4 et dans l'essai Prodige 23 par le biais d'une trithérapie [7]. Le Folfirinox est également proposé dans l'étude GRECCAR 12. Son bras expérimental comporte 4 cycles de chimiothérapie d'induction avant radio-chimiothérapie, comparé dans une étude de phase 3 randomisée avec la radio-chimiothérapie préopératoire. Le traitement par radio-chimiothérapie préopératoire comporte un CAP50 dans les deux bras de traitement (radiothérapie jusqu'à 50 Gy pour la tumeur rectale, et Capécitabine $1600 \mathrm{mg} / \mathrm{m}^{2} / \mathrm{j}$ de traitement par radiothérapie). L'objectif principal est de comparer le taux de préservation d'organe entre ces deux bras expérimentaux un an après la chirurgie. Les objectifs secondaires sont classiques. Les critères d'inclusion concernent des tumeurs classées cT2T3, N0 ou N1 (inférieures ou égales à 3 ganglions), de taille inférieure ou égale à $8 \mathrm{~cm}$, réduite à $4 \mathrm{~cm}$ pour des tumeurs localisées à moins de $10 \mathrm{~cm}$ de la marge anale. La chirurgie est programmée à la dixième semaine, avec une évaluation de la réponse tumorale à la huitième semaine après la fin du traitement néoadjuvant dans les deux bras. Une bonne réponse radiologique correspond à un résidu tumoral inférieur ou égal à deux centimètres. L'exérèse locale est préconisée chez les bons répondeurs. L'ablation totale du mésorectum est indiquée chez les mauvais répondeurs. Deux cent dix-huit patients sont attendus sur une période d'inclusion de 36 mois.

Toujours dans le domaine de la conservation sphinctérienne, l'essai OPERA s'attache à mesurer cet objectif à 3 ans lorsqu'est ajouté un traitement endo-cavitaire par radiothérapie de contact après un traitement standard par association radio-chimiothérapie pour les tumeurs classées T2, T2a ou T2b, N0 ou N1. Le bras standard comprend une radiothérapie externe délivrant 45Gy avec la Capécitabine suivie d'un boost de 9Gy en 5 fractions, comparé à un bras expérimental dans lequel, après les $45 \mathrm{~Gy}$ en 25 fractions, le complément local est adapté à la taille de la tumeur. Si la lésion résiduelle est de taille inférieure à $3 \mathrm{~cm}$, le complément de traitement est apporté par 3 fractions de 30Gy délivrées à la tumeur par radiothérapie de contact AVANT l'irradiation externe. Si la tumeur fait plus de $3 \mathrm{~cm}$, le complément de traitement est délivré APRÈS la radiothérapie externe, après une période de 2 semaines. Cette étude de phase III, qui a débuté en mars 2015, doit inclure 236 patients.

L'étude PROSPECT, conduite par le CNI, compare chez des patients porteurs de tumeurs classées T2N1, T3N0, T3N1 un bras standard comportant une association radio-chimiothérapie précédant une chirurgie classique suivie de chimiothérapie adjuvante par Folfox à un bras expérimental comportant une chimiothérapie par Folfox pour 12 semaines précédant une évaluation par IRM. Si 
la réponse est jugée supérieure à $20 \%$ sur l'IRM d'évaluation, le patient est ensuite conduit à la chirurgie sans radio-chimiothérapie préopératoire. Les malades à haut risque en situation postopératoire se verront proposer une radio-chimiothérapie post-opératoire. Cette étude a débuté en janvier 2012. Elle doit recruter 1060 patients et se terminer au cours de l'année 2017. L'analyse finale portant sur le taux de résection pelvienne $\mathrm{R} 0$, la survie sans maladie, le temps à récidive locale, la réponse pathologique complète et la survie globale est attendue dans 8 ans.

\section{TUMEURS LOCALEMENT AVANCÉES}

Les questions majeures posées par les études s'adressant à cette population sont de deux ordres : faire face à l'échec à distance qui devient prédominant dans cette population de patient et, quand elle est possible, contribuer à une conservation sphinctérienne fonctionnellement satisfaisante. Le standard fait appel à une chimio-radiothérapie associant Capécitabine et radiothérapie délivrant au minimum 45 Gy en 5 semaines. L'essai PRODIGE 23 compare cette radio-chimiothérapie préopératoire à une chimiothérapie par Folfirinox précédant la radio-chimiothérapie préopératoire chez des patients porteurs d'une maladie localement avancée rectale. L'objectif principal est basé sur la survie sans maladie, mesurée à 3 ans. Quatre cent soixante patients sont attendus dans cette étude qui a débuté en 2012 et dont l'arrêt des inclusions vient d'être prononcé. Les patients étaient porteurs de tumeurs classées cT3 ou cT4, considérées potentiellement résécables après radiochimiothérapie. L'autre approche, qui fait suite au GRECCAR 4, ayant testé l'induction par le Folfirinox puis la délivrance d'une association radio-chimiothérapie adaptée à la réponse tumorale volumétrique, est le protocole GRECCAR 14, étude prospective de phase 3 dont l'objectif principal est de comparer ce traitement personnalisé à une attitude standard préopératoire pour des patients porteurs de tumeurs classées T3c ou T3d, T4a ou T4b, quel que soit le status ganglionnaire avec une CRM prédictive inférieure à $2 \mathrm{~mm}$. Le bras expérimental dans une randomisation 2-1 teste l'apport de 6 cycles de Folfirinox avant une réévaluation par IRM et PET puis de proposer, en fonction de la réponse mesurée sur l'IRM chez les patients bons répondeurs avec une réponse supérieure à $60 \%$ et une CRM supérieure à $1 \mathrm{~mm}$, une chirurgie seule, sans radio-chimiothérapie préopératoire. Pour les patients du groupe favorable, après réponse estimée entre $40 \%$ et $60 \%$, le traitement comporte une radio-chimiothérapie délivrant 50Gy associée à la Capécitabine avant la chirurgie d'exérèse de type TME. Pour les patients du groupe défavorable, avec une réponse inférieure à $40 \%$ ou CRM inférieure à $1 \mathrm{~mm}$, la radio-chimiothérapie comporte un schéma CAP60 précédant la chirurgie. Le bras contrôle comporte une radio-chimiothérapie à la dose de 50Gy suivie d'une chirurgie. Le traitement systémique adjuvant est laissé à la discrétion de l'investigateur mais peut être clairement évité en cas de réponse complète ou pratiquement complète.

\section{ETUDES TRANSLATIONNELLES}

Ces études vont devoir être systématiquement associée aux études cliniques pour contribuer à évaluer avec la plus grande pertinence la réponse initiale des tumeurs aux traitements d'induction et pour déterminer avec précision les facteurs prédictifs de réponse. Parmi ces approches, l'étude RADON vient illustrer une de ces démarches. Il s'agit d'une étude observationnelle prospective, 
qui a pour but de déterminer pour les adénocarcinomes rectaux si la présence, le taux, et la cinétique de l'ADN (acide déoxyribonucléique) tumoral circulant, au diagnostic, et au décours de la chimioradiothérapie préopératoire, permettent de prédire la réponse à la chimioradiothérapie et la survie des patients. Cette approche est particulièrement intéressante en appui d'une stratégie de "watch and see" (surveillance sans chirurgie en cas de réponse clinique complète après chimioradiothérapie pré-opératoire). Les objectifs secondaires sont l'analyse de l'impact de certaines mutations connues, telles que KRAS et TP53, sur la chimiosensibilité et la radiosensibilité des tumeurs. Elle doit permettre de déterminer si la présence d'ADN tumoral circulant au diagnostic, après chimioradiothérapie préopératoire, après chirurgie et à distance est corrélée à la survie sans récidive locale, à l'extension de la maladie à distance, et à la survie globale.

Un prélèvement sanguin est proposé à tous les patients vus en consultation pour un cancer du rectum localement avancé (T3, T4, N+), à qui il est proposé un traitement par chimioradiothérapie préopératoire suivi d'un traitement chirurgical dans les services d'oncologie radiothérapie de l'Assistance Publique Hôpitaux de Paris (Hôpital Européen Georges Pompidou, Hôpital Henri Mondor, GHU La Pitié Salpêtrière Charles Foix, Hôpital Saint Louis, Hôpital Tenon). Les prélèvements sanguins sont réalisés à 4 temps différents de la prise en charge, en préthérapeutique, entre 1 et 4 semaines avant le début de la chimioradiothérapie, intermédiaire (à la fin du traitement par chimioradiothérapie), post-chirurgicale (1 mois après le traitement chirurgical) et au cours de la surveillance ( 6 mois après l'intervention chirurgicale). Les analyses d'ADN tumoral circulant sont réalisées par extraction d'ADN, recherche de mutations, analyse sur puces à haute densité et PCR digitale en gouttelettes.

\section{CONCLUSION}

Le traitement des cancers du rectum est toujours aussi passionnant pour l'oncologue radiothérapeute alors que le pronostic loco-régional semblait être un acquis définitif grâce à l'association chimio-radiothérapie préopératoire et la chirurgie d'exérèse du mésorectum. Un panel d'études thérapeutiques porte désormais sur la conservation du sphincter et sur le pronostic global de la maladie avec une personnalisation des traitements qui repose sur l'évaluation initiale de la maladie grâce à l'imagerie, l'étude de la réponse aux traitements d'induction par l'imagerie et la biologie. Ces essais ne concernent peu de nouvelles drogues ou thérapies ciblées qui ont jusqu'à ce jour déçues dans ces objectifs. 


\section{REFERENCES}

1-Allegra CJ, Yothers G, O'Connell MJ, Beart RW, Wozniak TF, Pitot HC et al. Neoadjuvant 5-FU or Capecitabine Plus Radiation With or Without Oxaliplatin in Rectal Cancer Patients: A Phase III Randomized Clinical Trial. J Natl Cancer Inst. 2015;107(11).

2-Aschele C, Cionini L, Lonardi S, Pinto C, Cordio S, Rosati G et al. Primary Tumor Response to Preoperative Chemoradiation With or Without Oxaliplatin in Locally Advanced Rectal Cancer: Pathologic Results of the STAR-01 Randomized Phase III Trial. J Clin Oncol. 2011;29:2773-80.

3-Borg C, André T, Mantion G, Boudghène F, Mornex F, Maingon P et al Pathological response and safety of two neoadjuvant strategies with bevacizumab in MRI-defined locally advanced T3 resectable rectal cancer: a randomized, noncomparative phase II study. Ann Oncol 2014;11:2205-10

4- Gérard JP, Azria D, Gourgou-Bourgade S, Martel-Lafay I, Hennequin C, Etienne PL et al Clinical outcome of the ACCORD 12/0405 PRODIGE 2 randomized trial in rectal cancer J Clin Oncol. 2012;30:4558-65

5-Habr-Gama A, Lynn PB, Jorge JM, São Julião GP, Proscurshim I, Gama-Rodrigues J, et al Impact of Organ-Preserving Strategies on Anorectal Function in Patients with Distal Rectal Cancer Following Neoadjuvant Chemoradiation. Dis Colon Rectum. 2016; 59:264-9

6-Machiels JP, Sempoux C, Scalliet P, Coche JC, Humblet Y, Van Cutsem E, et al Phase I/II study of preoperative cetuximab, capecitabine, and external beam radiotherapy in patients with rectal cancer. Ann Oncol. 2007;4:738-44.

7-Rouanet P, Rullier E, Lelong B, Maingon P, Tuech JJ, Pezet D, et al Tailored Treatment Strategy for Locally Advanced Rectal Carcinoma Based on the Tumor Response to Induction Chemotherapy: Preliminary Results of the French Phase II Multicenter GRECCAR4 Trial. Dis Colon Rectum. 2017 ;60:653-663

8-Rullier E, Rouanet P, Tuech JJ, Valverde A, Lelong B, Rivoire $\mathrm{M}$ et al. Organ preservation for rectal cancer (GRECCAR 2): a prospective, randomised, open-label, multicentre, phase 3 trial. Lancet. 2017;17:31056-5

9-São Julião GP, Ortega CD, Vailati BB, Habr-Gama A, Fernandez LM, Gama-Rodrigues J, et al Magnetic resonance imaging following neoadjuvant chemoradiation and transanal endoscopic microsurgery for rectal cancer. Colorectal Dis. 2017;6:196-203 